\title{
Extrauterine Neuromaturation of Low Risk Preterm Infants
}

\author{
MARILEE C. ALLEN, SUSAN AUCOTT, ELIZABETH A. CRISTOFALO, GREG R. ALEXANDER, AND PAMELA K. DONOHUE \\ Department of Pediatrics, Johns Hopkins School of Medicine, Baltimore, Maryland 21287 [M.C.A., S.A., E.A.C., P.K.D.]; and Department \\ of Pediatrics, University of South Florida, Tampa, Florida 33620 [G.R.A.]
}

\begin{abstract}
The objective of the study was to follow neuromaturation in preterm infants. From serial exams in 90 low risk very low birthweight infants, each infant's Maturity Scores (the sum of tone, reflex, and response items) were plotted against postmenstrual age (PMA) when examined. Each infant's estimated line of best fit provides two descriptors of that infant's neuromaturation: slope (Individual Maturity Slope) and $y$-value (Predicted Maturity Score at 32-wk PMA). We found that Maturity Scores increased with PMA; $96 \%$ had correlation coefficients $>0.8$. Mean Actual and Predicted Maturity Scores at 32-wk PMA were 60 and 58, respectively, in 65 infants. When stratified by gestational age, Mean Actual Maturity Score at 30 -wk PMA were 50 whether infants were 1 or several weeks old when examined. Therefore, low risk preterm infants demonstrated individual variability in rate of neuromaturation. Tone, reflexes, and responses nonetheless emerged in a predictable pattern, whether neuromaturation was intrauterine or extrauterine. This unique tool that measures preterm neuromaturation requires expertise but no technology. It has an exciting potential for providing insight into how emerging central nervous system function and structure influence each other, as well as how the central nervous system recovers from injury. (Pediatr Res 65: 542-547, 2009)
\end{abstract}

$\mathrm{N}^{\mathrm{cos}}$ euromaturation is the functional development of the central nervous system (CNS) and results from continuous interactions between the genome and the environment of the fetus, infant and child. Gesell, André-Thomas, Amiel-Tison, and many others have described the stages of neuromaturation in the fetus, preterm infant, full-term neonate and young child (1-19). Understanding the typical pattern of infant neuromaturation provides a framework for recognizing atypical patterns from CNS injury as early as possible. Despite increasing sophistication of neuroimaging technology, evaluation of CNS function is still necessary for diagnosing neurodevelopmental disability. Early diagnosis currently relies on assessing infant function: achievement of gross motor milestones (e.g. rolling, sitting, walking) reflects an infant's emerging control over posture and movement as well as inhibition of the flexor hypertonia, hyperreflexia, and pathologic and primitive reflexes characteristic of infants at term $(11,12,14,20,21)$.

Received March 17, 2008; accepted December 1, 2008.

Correspondence: Marilee C. Allen, M.D., The Johns Hopkins Hospital, Nelson 2-133, 600 N. Wolfe St., Baltimore, MD 21287-3200; e-mail: mallen2@jhmi.edu

Supported by grant R40 MC 00312 from the Maternal and Child Health Bureau (Title V, Social Security Act), Health Resources and Services Administration, Department of Health and Human Services.

G.R.A. is deceased.
In conjunction with neuroimaging studies, comprehensive neurodevelopmental examinations of preterm infants help us to estimate degree of risk for neurodevelopmental disabilities, so that we can better support their development in the neonatal intensive care unit (NICU), counsel their parents, and plan for community services after discharge (22-24). The higher the number and severity of neuromotor abnormalities, the higher the risk of cerebral palsy, cognitive impairment, and minor neuromotor dysfunction (25). As recognized by Amiel-Tison, serial examinations helps determine which abnormalities persist $(5,26)$.

The growth, maturation, and myelination of sensorimotor cortical pathways are reflected in dramatic but predictable changes in muscle tone, movements, reflexes, and responses with increasing postmenstrual age (PMA, chronologic age plus the infant's gestational age at birth) $(5,8,26-$ 28). Development before term is characterized not by attainment of discrete milestones but by a gradual emergence of flexor extremity tone, axial tone (neck, trunk, shoulders, and hips), deep tendon reflexes, pathologic reflexes (e.g. Babinski), primitive reflexes (e.g. Moro), postural head control, and sensory responses $(1-3,22)$. As a method of following neuromaturation of preterm infants in our NICU, we devised a Maturity Score for each exam by summing examination items graded to reflect the successive increase in strength and completeness of responses with PMA.

Recognizing the difficulty of describing "normal" extrauterine development before term, this article describes Maturity Scores in a sample of preterm infants with no evidence of major risk factors for neurodevelopmental disability or factors that influence early neurodevelopment. Being able to measure early CNS function can improve our ability to counsel individual families about their infants. Providing earlier feedback regarding preterm neuromaturation would enhance safety in randomized clinical trials in the NICU. Our measures of early preterm neuromaturation have the exciting potential to provide insight into the complex relationship between emerging CNS function and CNS structure, and to provide insight into how the developing CNS recovers from injury.

\footnotetext{
Abbreviations: CLD, chronic lung disease, PMA, postmenstrual age in weeks, gestational age plus chronological age, VLBW, very low birthweight, with birthweight below $1500 \mathrm{~g}$
} 


\section{METHODS}

Study design. This observational cohort study prospectively tracked the early development of neuromotor abnormalities in infants with birthweights below $1500 \mathrm{~g}$ in our tertiary care NICU and was approved by the Johns Hopkins Medicine Institutional Review Boards. From 1994 to 2000, a single experienced examiner (M.C.A., a neonatologist and neurodevelopmental pediatrician) performed weekly neonatal neurodevelopmental examinations on over 500 very low birthweight (VLBW) infants born at or transferred within 2 wk to our NICU (25). This article focuses not on neuromotor abnormalities but on tracking neuromaturation in a subset of 90 low risk preterm infants by plotting their Maturity Scores against their PMA at the time of the exam. Gestational age was determined by best obstetrical estimate, which included first trimester ultrasound when available. The change in Maturity Scores with PMA in this low risk VLBW sample represents extrauterine neuromaturation before term. For each individual infant, we used the slope of an estimated line of best fit (Individual Maturity Slope) and $y$-value at a selected PMA (e.g. Predicted Maturity Score at 32-wk PMA) to denote that infant's rate and degree of neuromaturation, respectively.

Study sample. Preterm infants with birthweights below $1500 \mathrm{~g}$ who survived to NICU discharge were hospitalized at least $3 \mathrm{wk}$ (to allow for 3 or more weekly exams) and had no significant risk factors for neurodevelopmental disability were included. Based on preterm outcomes literature $(29,30)$, the following were the exclusion criteria: intracranial hemorrhage, hydrocephalus, ventricular dilation, intraparenchymal cysts, seizures, Apgar score below 4 at $5 \mathrm{~min}$, cord $\mathrm{pH}$ below 7.15, major congenital anomalies, intrauterine growth restriction (IUGR), chronic lung disease, and exposure to illicit drugs. We include two infants with familial polydactyly and one infant with a hydrocoele. Criteria for IUGR were strict: birthweight at or below 10 percentile for gestational age (GA) using race-specific and gender-specific norms (31). We defined chronic lung disease as requiring oxygen beyond $28 \mathrm{~d}$ (32). Because hypertonia has been described with prenatal exposure to narcotics, we excluded infants with positive maternal history of cocaine or narcotics or positive urine toxicology screens from mother or infant (33).

Neonatal neurodevelopmental examination. Our comprehensive neonatal neurodevelopmental examination draws from the work of Capute, AndréThomas, Saint-Anne Dargassies, Amiel-Tison, Illingworth, Paine, Prechtl, Peiper, Robinson, Dubowitz, and Brazelton $(6,7,9-11,15,16,18,19,34)$. Many exam items assess the emergence of extremity flexor tone, axial (neck, trunk, shoulder, and hip) tone, deep tendon reflexes, pathologic reflexes, primitive reflexes, postural head control, sensory responses, and behavior (Table 1). In a sample of preterm infants who did not develop cerebral palsy, we demonstrated a progressive increase in scores of many of these exam items from 26 to 40-wk PMA (1-3). A measure of degree of neuromaturation at a specific point in time, the Maturity Score, is the sum of item scores, and ranges from 0 to 150.

The Maturity Score has three component parts: Tone Subscore + Reflex Subscore + Responses $=$ Maturity Score. The Tone Subscore consists of individual examination items that evaluate degree of extremity flexor tone and axial tone. The Reflex Subscore is composed of items that measure strength of the deep tendon reflexes, pathologic reflexes (Babinski, Chaddocks, Mass Reflex, and Crossed Adduction), and 10 primitive reflexes scored on a scale of $0-4$ in the manner of Capute et al. $(2,21)$. The primitive reflexes include the Moro, upper and lower extremity grasp reflexes, asymmetric tonic neck reflex, Galant, lower extremity placing, stepping, tonic labyrinthine, symmetric tonic neck reflex, and positive support. Response items include visual fixation, visual following, and postural head control $(5,25,26)$.

Graphic representation of neuromaturation. Comparing Actual Maturity Scores in groups of infants requires examining them all at the exact same PMA, which is not feasible in a busy NICU. Examinations were deferred in sick infants and infants unable to tolerate handling. The need to aggregate data on individual infants for group comparisons led us to graphic representations of an individual infant's neuromaturation, by plotting the infant's Maturity Scores against PMA at the time of the exam (Figs. 1 and 2). On a case-by-case basis, a computer program [SPSS (35)] estimates a line of best fit for each infant's scatterplot. The slope of the estimated line of best fit, or Individual Maturity Slope, reflects that infant's rate of neuromaturation before term. The Individual Maturity Slope value for the infant in Figure 1 is 7.4, and for the infant in Figure 2 , it is 6.9. Although we explored $y$-values at 30,32 , and 34-wk PMA, we chose 32-wk PMA for comparing degree of neuromaturation based on its clinical significance. By 32-wk PMA, most preterm infants have less apnea, less need for temperature support, and have begun to coordinate sucking, swallowing, and breathing. Both the Individual Maturity Slope value and the Predicted Maturity Score at 32-wk PMA represent individual level data. By describing an individual preterm infant's rate and degree of
Table 1. Components of neonatal neurodevelopmental examination

\begin{tabular}{ll}
\hline \multicolumn{1}{c}{ Category } & \multicolumn{1}{c}{ Examples } \\
\hline Deep tendon reflexes & Upper extremity, knee, ankle \\
Pathologic reflexes & Babinski \\
Clonus & Absent, intermittent, or sustained \\
Extremity tone & Popliteal angle, recoil \\
Head and neck control & Pull to sit \\
Truncal tone & Ventral suspension \\
Primitive reflexes & Galant, moro, righting reaction \\
Cranial nerve/oromotor function & Root, suck \\
Posture & Extension, semiflexion, flexion \\
& of extremities \\
Spontaneous movements & Hand movements, extremity \\
& movement \\
Visual sensory responses & Fixation, tracking \\
Behavior & Crying, lethargy, jitteriness \\
\hline
\end{tabular}

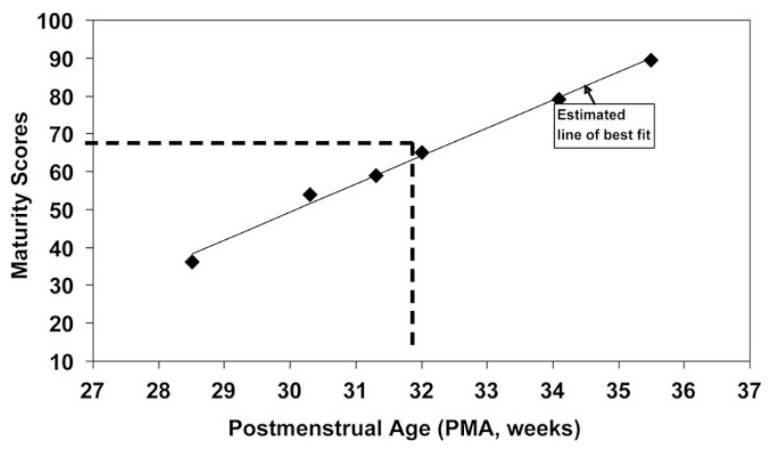

Figure 1. Maturity scores for an infant born at 27-wk gestation. Actual and Predicted Maturity Score at 32-wk PMA are both 65. Individual maturity slope is 7.4. The dotted line demonstrates determination of Predicted Maturity Score as the $y$-intercept at 32-wk PMA from the line of best fit.

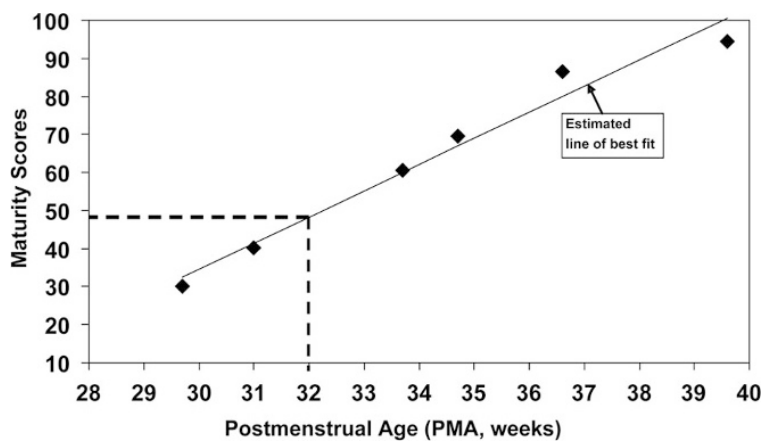

Figure 2. Maturity Scores for an infant born at 28 5/7 wk gestation. Predicted Maturity Score at 32-wk PMA is 49. Individual maturity slope is 6.9. The dotted line demonstrates determination of Predicted Maturity Score at 32-wk PMA from the line of best fit.

neuromaturation in the NICU, these clinical measures can be used to compare an individual infant to a group and to compare groups of infants.

The correlation coefficient $(r)$ measures how well an individual infant's Maturity Scores fit the estimated line of best fit. To emphasize that this $r$ represents individual level data, we designate it the Individual Correlation Coefficient, with 1.0 representing a perfect linear fit of Maturity Scores to the line of best fit. In Figures 1 and 2, the Individual Correlation Coefficients were 0.99 and 0.98 , respectively.

Data analyses. For each infant, we used SPSS to plot Maturity Scores against PMA, estimate a line of best fit, and determine that infant's Individual Maturity Slope, Predicted Maturity Score at 32-wk PMA, and Individual Correlation Coefficient values. Means and confidence intervals were calculated for sample characteristics, examination factors, and Maturity Score descriptors (Tables 1 and

2). We evaluated the distributions of values for Individual Maturity Slope and 
Table 2. Characteristics of low risk preterm infants and their examinations

\begin{tabular}{lc}
\hline \multicolumn{1}{c}{ Characteristic } & $\begin{array}{c}\text { Mean } \\
(95 \% \text { confidence intervals }) \\
\text { or } N(\%)\end{array}$ \\
\hline Birthweight (BW) & $1159 \mathrm{~g}(1123-1194)$ \\
Gestational age (GA) & $28.8 \mathrm{wk}(28.5-29.0)$ \\
Gender-males & $41(46)$ \\
Race & \\
African American & $57(63)$ \\
White & $31(34)$ \\
Other & $2(2)$ \\
Multiple gestation & $23(26)$ \\
Outborn (transferred within 2 wk) & $6(7)$ \\
Mode of delivery-vaginal & $54(60)$ \\
Antenatal steroids & $67(74)$ \\
Duration of ventilation & $2 \mathrm{~d}(1-3)$ \\
Duration of oxygen & $7 \mathrm{~d}(5-9)$ \\
Age when regained birthweight & $16 \mathrm{~d} \mathrm{(15-17)}$ \\
Number of examinations & $5.5(5.0-5.9)$ \\
Postmenstrual age at first exam & $29.9 \mathrm{wk}(29.7-30.2)$ \\
Postmenstrual age at last exam & $34.9 \mathrm{wk}(34.4-35.3)$ \\
\hline
\end{tabular}

Predicted Maturity Score values (e.g. scatterplots, boxplots, KolmogorovSmirnov test with Lillienfors correction, skewness, and kurtosis). Tone and Reflex Subscores were similarly evaluated. We expected Individual Correlation Coefficient values to be skewed toward 1.0, so medians and $\mathrm{M}$ estimators per Hampel are reported. For infants who had neonatal neurodevelopmental examinations at 32-wk PMA, the correlation between their Actual and Predicted Maturity Scores at 32-wk PMA was determined.

To compare extrauterine with intrauterine neuromaturation, we used independent $t$ test to compare Actual Maturity Scores at 30-wk PMA for two groups: 1) infants born at or before 28 wk gestation who had one or more previous examinations and 2) infants born at $29 \mathrm{wk}$ gestation examined at 1 wk chronological age (at 30-wk PMA).

\section{RESULTS}

Study sample. Table 2 presents characteristics of the 90 VLBW infants who met low risk study criteria. Mean birthweight was 1159 (95\% confidence interval, 1123-1194) g and mean GA was 28.8 (28.5-29.0) wk. Twenty-four percent of the sample was born at 25 through 27 completed weeks gestation, $58 \%$ at 28 through 29 wk, and $18 \%$ at 30 through 31 wk. The mean number of neonatal neurodevelopmental examinations per child was $5.5(5.0-5.9)$, and ranged from 3 to 11 exams. The number of examinations reflected the infant's GA, severity of illness and length of hospital stay.

Actual maturity scores. For the infant represented in Figure 1, born at 27 wk gestation, both his Predicted Maturity Score at 32-wk PMA (derived from his line of best fit) and his Actual Maturity Score (calculated from his exam at 32-wk PMA) are 65. For the infant represented in Figure 2, who was born at $285 / 7$ wk but not examined at 32-wk PMA, his Predicted Maturity Score at 32-wk PMA of 49 can be used for comparisons.

Figure 3 is a scatterplot of all Actual Maturity Scores by each week PMA. Actual Maturity Scores at each week PMA, from 28 to 39-wk PMA, were normally distributed. (There were fewer than 10 exams for each week of PMA before 28 wk and after 37 wk.) Mean Actual Maturity Scores are plotted for each week of PMA, from 28 to 37 wk, in Figure 4.

Descriptors of neuromaturation. For each low risk preterm infant, Maturity Scores were easily calculated and increased

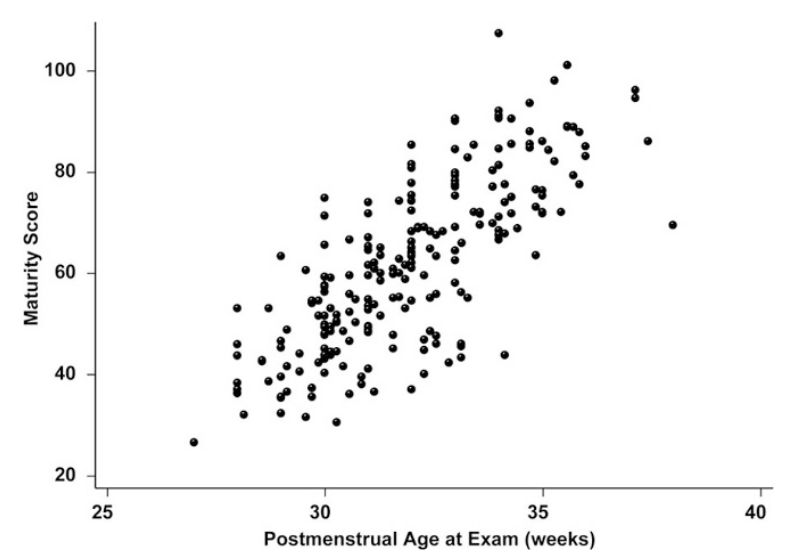

Figure 3. Scatterplot of Actual Maturity Scores plotted by week of PMA for 90 low risk preterm infants.

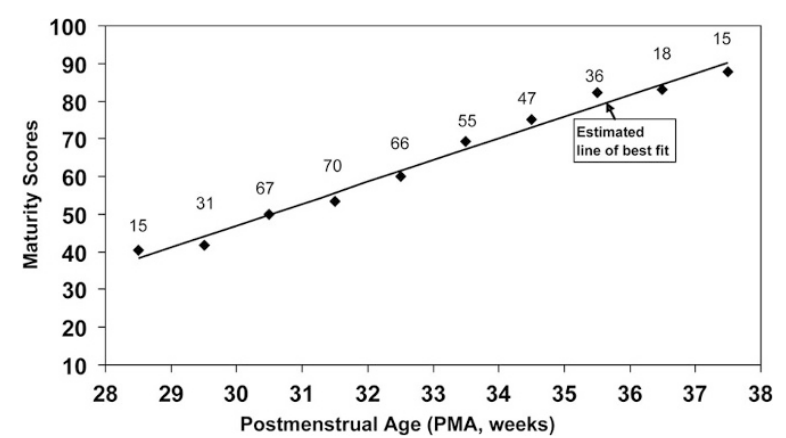

Figure 4. Mean Actual Maturity Scores for 90 low risk preterm infants, from 28 to 37-wk PMA. The numbers above each point indicates the number of infants examined at that week of PMA. A line of best fit is estimated from mean Maturity Scores for each week PMA.

Table 3. Summary statistics for descriptors of neuromaturation in 90 low risk preterm infants

\begin{tabular}{lc}
\multicolumn{1}{c}{ Descriptor } & $\begin{array}{c}\text { Mean } \\
(95 \% \text { confidence intervals })\end{array}$ \\
\hline Individual maturity slope & $8.0(7.4-8.5)$ \\
Predicted Maturity Score at & $60(58-62)$ \\
32-wk PMA & \\
Individual tone slope & $3.6(3.3-3.9)$ \\
Tone Subscore at 32-wk PMA & $19(17-20)$ \\
Individual reflex slope & $4.0(3.7-4.4)$ \\
Reflex Subscore at 32-wk PMA & $34(33-36)$ \\
\hline
\end{tabular}

linearly with PMA (as in Figs. 1 and 2). The group mean Individual Maturity Slope value was 8.0 (7.4-8.5) (Table 3). For the total sample, mean Predicted Maturity Score values at 30,32 , and 34 wk were 44 (42-46), 60 (58-62), and 76 (73-78), respectively. Values for Individual Maturity Slope and all three $y$-intercepts were normally distributed, with no skew or kurtosis. Table 3 provides similar statistics for Individual Tone and Reflex Subscore Slopes and Predicted Tone and Reflex Subscore values at 32-wk PMA.

Individual correlation coefficient values. The Individual Correlation Coefficient values (measuring degree of fit to the line of best fit) were above 0.80 in 86 infants $(96 \%$ of the sample). Only one infant's Individual Correlation Coefficient was below 0.60. Individual Correlation Coefficient values were skewed toward 1.0. The group mean Individual Corre- 
lation Coefficient value was 0.93 (0.91-0.95), but median and Hampel's M-Estimator were each 0.96. Median Individual Correlation Coefficient values for Tone and Reflex Subscores were 0.90 (0.88-0.92) and 0.90 (0.87-0.93), respectively.

Comparison of Actual Maturity Scores with Predicted Maturity Score values. For 65 infants who were examined at 32-wk PMA, Actual Maturity Scores were 60 (55-63), whereas Predicted Maturity Scores were 58 (54-60). Paired Sample Correlation was 0.88 .

Comparison of intrauterine with extrauterine neuromaturation. Of the GA categories in this sample, the largest group $(n=25)$ were infants born at 29 wk gestation and had neonatal neurodevelopmental examinations at 30-wk PMA. There was not a significant difference in the mean Actual Maturity Score values at 30-wk PMA when comparing 25 infants born at $29 \mathrm{wk}$ gestation examined in a week at 30-wk PMA with 42 infants born at or before 28 wk gestation examined at 30-wk PMA: 50 (47-53) versus 50 (46-53), respectively.

\section{DISCUSSION}

A progressive increase in Maturity Scores with PMA in these low risk preterm infants reflects the emergence of muscle tone, reflexes and responses described by Gesell, SaintAnne Dargassies, Amiel-Tison, Peiper, and others $(5,6,13,19,36)$. A linear increase with PMA in our low risk preterm infants confirms Madame Suzanne Saint-Anne Dargassies assertion that "successive maturational transformations are orderly, and progressively and gradually enriched: at identical stages of development, neurologic assessments are strictly comparable. Thus, maturative phenomena are intimately and exclusively a function of age" (19), p 227).

Just as older infants demonstrate individual variation in milestone attainment (12), these low risk preterm infants demonstrated individual variation in extrauterine neuromaturation: their Actual Maturity Scores, Individual Maturity Slope values, and Predicted Maturity Score at 32-wk PMA values were normally distributed. Individual variability of these measures is expected, as neuromaturation is the result of multiple gene-environmental interactions (including both the intrauterine and extrauterine environments). Maturity Scores provide a method for tracking an individual preterm infant's neuromaturation and comparing that infant to others. For example, a Maturity Score of 30 reveals hypotonia, minimal deep tendon reflexes, no head or trunk tone, no oromotor skills, flailing movements, and no visual fixation. In contrast, at 60 there are consistent deep tendon reflexes, mild resistance in extremity tone, minimal head and trunk tone, early oromotor function, jerky movements, intermittent flexion postures, and brief visual fixation. At 90, one sees brisk deep tendon reflexes, moderate extremity tone with emerging head control, uncoordinated oromotor skills, smooth and harmonious movements, increasing flexion and visual fixation with limited tracking. Although we cannot measure "normal" extrauterine development before term, this low risk preterm sample approximates "typical" extrauterine neuromaturation.
Aside from insults or injury, neuromaturation proceeds according to age in both intrauterine and extrauterine environments. Actual Maturity Scores at 30-wk PMA were the same whether measured $1 \mathrm{wk}$ after birth in infants born at $29 \mathrm{wk}$ gestation or after several weeks in infants born before 29 wk gestation. This finding is consistent with preterm development at term and during the first year. When neurologic assessments are compared, preterm infants at term are more similar to fullterm neonates than they are different (22). After term, healthy preterm infants attain motor milestones on the same timetable as fullterm infants, as long as their age is corrected for degree of prematurity $(20,22,37-39)$.

In comparison with other neonatal assessments, a unique aspect of our approach is its ability to capture the dynamic nature of neurodevelopment by quantifying degree of neuromaturation at a specific point in time and measuring rate of neuromaturation (40). It requires expertise but no technology. Although the Individual Maturity Slope value denotes an individual infant's rate of neuromaturation in the NICU, the Predicted Maturity Score at 32-wk PMA reflects both degree of neuromaturation at birth and rate of neuromaturation. We chose 32-wk PMA as a reflection of the transition from neonatal intensive care to convalescent care: by 32-wk PMA, most preterm infants begin to coordinate suck, swallow and breathe enough to try feeding, maintain their body temperatures enough to wean from an isolette, and have less apnea. The similarity between Actual and Predicted Maturity Scores at 32-wk PMA (60 versus 58, respectively, on a scale of $0-150)$ reassures us of its validity.

Funded by the Maternal and Child Health Bureau, a prospective study is in progress to evaluate how, in combination with quantification of neuromotor abnormalities and neuroimaging abnormalities, our measures of neuromaturation predict 18 mo neurodevelopmental outcome. With training, we have achieved good interrater reliability among neonatologists, neonatology fellows, and physician assistants. In this low risk sample, the linear increase and normal distribution of Maturity Scores with PMA indicate a sufficient number of examination items with wide enough ranges to adequately track preterm neuromaturation to approximately 35-wk PMA (Figs. 2-4). However, Maturity Scores tended to plateau beyond 35-wk PMA. The addition of some examination items from AmielTison's fullterm newborn examination to make our exam more robust at and beyond term $(5,8)$ will allow us to compare Actual and Predicted Maturity Scores at term.

There was one infant in this low risk sample with a low Individual Correlation Coefficient of 0.34. This infant was born at $291 / 7$ wk gestation and had only three examinations (our minimum). His clinical course in the NICU was characterized by feeding intolerance, ileus, and suspected sepsis. His Actual Maturity Score on his second exam (while recovering at 31-wk PMA) was 7 points lower than on his initial examination but recovered by his subsequent exam at $322 / 7$ wk PMA. This finding raises important questions regarding the effect of neonatal illness on our Maturity Score. Using our measures of neuromaturation to compare samples with and without neonatal illness and exploring differences in Tone and Reflex Subscores may provide insight into whether lower 
Maturity Scores reflect delayed neuromaturation or are merely artifacts of acute neonatal illness (40). Chronic intrauterine stress (e.g. preeclampsia, IUGR) can accelerate intrauterine neuromaturation $(40,41)$.

Our ongoing prospective study will evaluate our measures of neuromaturation in preterm infants with prenatal, perinatal, and neonatal illness. Reliable, valid measures of early CNS function have the exciting potential to be used in a variety of ways: 1) to help predict an individual infant's risk of neurodevelopmental disability, which facilitates parent counseling and more efficient utilization of often limited comprehensive follow-up and early intervention resources; 2) to provide insight into how intrauterine factors, neonatal illness, and complications of prematurity influence early CNS functional development (leading to new preventive and treatment strategies); 3) to analyze how CNS structure influences CNS function in preterm infants in a NICU; 4) to evaluate how preterm infants with CNS injury recover function (and develop new treatment strategies); and 5) to provide more rapid feedback regarding efficacy and side effects of obstetric and neonatal treatments on early CNS function. Many commonly used medications have not been fully evaluated as to their safety or efficacy in preterm infants $(42,43)$. As preventive and treatment strategies for CNS injury in preterm infants emerge, measures of early CNS function can assist in targeting subjects and improving safety in randomized controlled clinical trials.

The very concept of preterm birth involves organ maturation, but our understanding of the consequences of preterm birth is hindered by the paucity of measures of maturation in preterm infants. Because of individual variation in growth, development and the intrauterine, and extrauterine environments, neither pregnancy duration (i.e. gestational age) nor fetal/infant size (e.g. birthweight) adequately reflect organ vulnerability to preterm delivery or predict the toll of preterm exposure to the extrauterine environment. Neuromaturation is especially critical for determining the quality of extrauterine life for the child born preterm: the most immature infant is vulnerable to significant brain injury with resulting neurodevelopmental impairment and disability (40). Evaluation of the interrelationships between duration of pregnancy, fetal size and maturation, can provide insight into some of the factors that contribute to preterm birth (44).

In conclusion, serial neonatal neurodevelopmental examinations in preterm NICU infants require expertise but no technology, yet can quantify rate and degree of neuromaturation as well as persistence and severity of neuromotor abnormality (25). These low risk preterm infants demonstrated predictable neuromaturation according to age, confirmed the similarities of intrauterine and extrauterine neuromaturation timetables, and provide normative data regarding individual variability in neuromaturation of "typical" preterm infants. The ability to measure CNS function in preterm infants in a NICU has the exciting potential of providing greater insight into the relationships between early CNS structure and function, mechanisms of CNS injury and recovery from CNS injury.
Acknowledgments. Analytical clinical research is meaningless if one cannot interpret the data that have been gathered. For this, our research team relied heavily on the insights, the expertise, and the wisdom of coauthor and perinatal epidemiologist Greg Alexander, who died February 20, 2007.

\section{REFERENCES}

1. Allen MC, Capute AJ 1986 Assessment of early auditory and visual abilities of extremely premature infants. Dev Med Child Neurol 28:458-466

2. Allen MC, Capute AJ 1986 The evolution of primitive reflexes in extremely premature infants. Pediatr Res 20:1284-1289

3. Allen MC, Capute AJ 1990 Tone and reflex development before term. Pediatrics 85:393-399

4. Amiel-Tison C, Gosselin J 2001 Neurologic Development from Birth to Six Years. Baltimore: Johns Hopkins University Press, pp 1-125

5. Amiel-Tison C 2002 Clinical assessment of the infant nervous system. In: Levene MI, Chervenak FA, Whittle MJ, Bennett MJ, Punt J (eds) Fetal and Neonatal Neurology and Neurosurgery. London: Churchill Livingstone, pp 99-120

6. Amiel-Tison C 1968 Neurological evaluation of the maturity of newborn infants. Arch Dis Child 43:89-93

7. Amiel-Tison C, Grenier A 1986 Neurologic Assessment During the First Year of Life. New York: Oxford University Press, pp 1-197

8. Amiel-Tison C, Maillard F, Lebrun F, Breart G, Papiernik E 1999 Neurological and physical maturation in normal growth singletons from 37 to 41 weeks' gestation. Early Hum Dev 54:145-156

9. Andre-Thomas Chesni Y, Saint-Anne Dargassies S 1960 The Neurological Examination of the Infant. Little Club Clinics in Developmental Medicine No. 1. London: National Spastics Society, pp 1-50

10. Brazelton TB 1973 Neonatal Behavioral Assessment Scale. Philadelphia: J.B. Lippincott, pp 1-66

11. Capute AJ, Shapiro BK, Accardo PJ, Wachtel RC, Ross A, Palmer FB 1982 Motor functions: associated primitive reflex profiles. Dev Med Child Neurol 24:662-669

12. Capute AJ, Shapiro BK, Palmer FB, Ross A, Wachtel RC 1985 Normal gross motor development: the influences of race, sex and socio-economic status. Dev Med Child Neurol 27:635-643

13. Gesell AL, Amatruda CS 1945 The Embryology of Behavior. New York: Harper \& Brothers, pp 1-274

14. Illingworth RS 1972 The Development of the Infant and Young Child—Normal and Abnormal. Baltimore: The Williams and Wilkins Co., pp 1-377

15. Paine RS, Brazelton TB, Donovan DE, Drorbaugh JE, Hubbell JP Jr, Sears EM 1964 Evolution of postural reflexes in normal infants and in the presence of chronic brain syndromes. Neurology 14:1036-1048

16. Peiper A 1963 Cerebral Function in Infancy and Childhood. New York: Consultants Bureau Enterprises, pp 1-683

17. Prechtl HF 1997 State of the art of a new functional assessment of the young nervous system. An early predictor of cerebral palsy. Early Hum Dev 50:1-11

18. Prechtl HF 1977 The Neurological Examination of the Full-term Newborn Infant London: S.I.M.P. with William Heinemann Medical Books, pp 1-68

19. Saint-Anne Dargassies S 1977 Neurological Development of the Full-Term and Premature Neonate. Amsterdam: Elsevier/North-Holland Biomedical Press, pp $1-323$

20. Allen MC, Alexander GR 1992 Using gross motor milestones to identify very preterm infants at risk for cerebral palsy. Dev Med Child Neurol 34:226-232

21. Capute AJ, Palmer FB, Shapiro BK, Wachtel RC, Ross A, Accardo PJ 1984 Primitive reflex profile: a quantitation of primitive reflexes in infancy. Dev Med Child Neurol 26:375-383

22. Allen MC 2008 Preterm development. In: Accardo PJ (ed) Capute and Accardo's Neurodevelopment in Infancy and Childhood. Baltimore: Paul H. Brookes Publishing Co, pp 29-45

23. Allen MC 2008 The neonatal neurodevelopmental examination. In: Accardo PJ (ed) Capute and Accardo's Neurodevelopmental Disabilities in Infancy and Childhood. Baltimore: Paul H. Brooke Publishing Co., pp 333-365

24. Aucott S, Donohue PK, Atkins E, Allen MC 2002 Neurodevelopmental care in the NICU. Ment Retard Dev Disabil Res Rev 8:298-308

25. Allen MC, Capute AJ 1989 Neonatal neurodevelopmental examination as a predictor of neuromotor outcome in premature infants. Pediatrics 83:498-506

26. Gosselin J, Gahagan S, Amiel-Tison C 2005 The Amiel-Tison neurological assessment at term: conceptual and methodological continuity in the course of follow-up. Ment Retard Dev Disabil Res Rev 11:34-51

27. Sarnat HB 1989 Do the corticospinal and corticobulbar tracts mediate functions in the human newborn. Can J Neurol Sci 16:157-160

28. Sarnat HB 1984 Anatomic and physiologic correlates of neurologic development in prematurity. In: Sarnat HB (ed) Topics in Neonatal Neurology. New York: Grune and Stratton, pp 1-24

29. Allen MC 2004 Risk assessment and neurodevelopmental outcomes. In: Taeusch HW, Ballard R, Gleason CA (eds) Avery's Diseases of the Newborn. Philadelphia: W.B. Saunders Co., pp 1026-1042

30. Allen MC 2002 Preterm outcomes research: a critical component of neonatal intensive care. Ment Retard Dev Disabil Res Rev 8:221-233

31. Alexander GR, Himes JH, Kaufman RB, Mor J, Kogan M 1996 A United States national reference for fetal growth. Obstet Gynecol 87:163-168 
32. Ehrenkranz RA, Walsh MC, Vohr BR, Jobe AH, Wright LL, Fanaroff AA, Wrage LA, Poole K 2005 Validation of the National Institutes of Health consensus definition of bronchopulmonary dysplasia. Pediatrics 116:1353-1360

33. Frank DA, Augustyn M, Knight WG, Pell T, Zuckerman B 2001 Growth, development, and behavior in early childhood following prenatal cocaine exposure: a systematic review. JAMA 285:1613-1625

34. Dubowitz L, Dubowitz V 1981 The Neurological Assessment of the Preterm and Fullterm Infant. Philadelphia: J. B. Lippincott Co., pp 1-103

35. SPSS for Windows Rel. 14.0 2005. Chicago: SPSS Inc

36. Amiel-Tison C 1976 A method for neurologic evaluation within the first year of life. Curr Probl Pediatr 7:1-50

37. Allen MC, Alexander GR 1990 Gross motor milestones in preterm infants: correction for degree of prematurity. J Pediatr 116:955-959

38. Den Ouden L, Rijken M, Brand R, Verloove-Vanhorick SP, Ruys JH 1991 Is it correct to correct? Developmental milestones in 555 "normal" preterm infants compared with term infants. J Pediatr 118:399-404
39. Palisano RJ 1986 Use of chronological and adjusted ages to compare motor development of healthy preterm and fullterm infants. Dev Med Child Neurol 28:180-187

40. Allen MC 2005 Assessment of gestational age and neuromaturation. Ment Retard Dev Disabil Res Rev 11:21-33

41. Amiel-Tison C, Cabrol D, Denver R, Jarreau PH, Papiernik E, Piazza PV 2004 Fetal adaptation to stress. I. acceleration of fetal maturation and earlier birth triggered by placental insufficiency in humans. Early Hum Dev 78:15-27

42. Finer NN, Higgins R, Kattwinkel J, Martin RJ 2006 Summary proceedings from the apnea-of-prematurity group. Pediatrics 117:S47-S51

43. Walsh MC, Szefler S, Davis J, Allen M, Van ML, Abman S, Blackmon L, Jobe A 2006 Summary proceedings from the bronchopulmonary dysplasia group. Pediatrics 117:S52-S56

44. Institute of Medicine Committee on Understanding Premature Birth and Assuring Healthy Outcomes 2006 IOM Preterm Birth: Causes, Consequences and Prevention. Washington, DC: National Academies Press 